\title{
INTRODUCTION \\ WRESTLING WITH BIOMEDICAL RESEARCH RESULTS: LANGUAGE RESOURCES AND LITERATURE ANALYSIS
}

\author{
GUEST EDITORS: \\ D. REBHOLZ-SCHUHMANN, NIGEL COLLIER, \\ JONG C. PARK and LIMSOON WONG
}

\begin{abstract}
Biomedical research spans from experimental molecular biology to genetic screening of selected populations (1000 genomes project) analyzing processes in core biology or delivering results to novel medical applications. These research domains are further augmented by research in the chemical domain with relevance to molecular biology and by research from the simulation of physiological and biochemical processes.
\end{abstract}

All involved research domains make use of computer-based solutions to define their knowledge space using publicly available data resources. For example, annotated facts are gathered and delivered in specialized databases; ontologies are being built to provide electronic access to terminological and conceptual knowledge and both types of resources are brought together to analyze the scientific literature for novel facts. We can expect for the future that all scientific biomedical information will be delivered through interfaces that seamlessly integrate the facts from all domains into one coherent knowledge space. ${ }^{1}$

Participants of "The 3rd International Symposium on Languages in Biology and Medicine" (LBM2009) contribute with their research work to these research goals. This issue contains three publications from LBM2009 that report on scientific results leading to better integration of scientific database resources and the scientific literature. This integration process requires standards for the automatic processing of literature resources, measurements on the achievements of the integration process and large-scale language resources to bridge the different scientific domains. All these developments support the building of the knowledge space spanning from biology to medicine through chemistry, biochemistry and physiology.

Contributions to LBM2009 that are relevant to researchers in molecular biology are presented in this issue, and other contributions that focus on the exploitation or construction of semantic resources will be provided through the Journal of Biomedical Semantics. The publication of Miwa et al. ${ }^{2}$ demonstrates results on the 
extraction of complex events such as genetic regulation from the scientific literature. The analytical method selects features and simple events from the text and then composes the complex events out of the findings. Sasaki et al. ${ }^{3}$ measure the improvement of information retrieval methods upon integration of a large-scale terminological resource into their solutions. The performance can be improved, but does not reach the best performing solutions in the competition. Rebholz-Schuhmann et al. ${ }^{4}$ report on a novel approach to generate a large-scale annotated corpus by combining annotation services from different sites (CALBC challenge). This approach would lead to the first annotated corpus that covers biological, chemical and medical terms at a significant scale and enables the development of annotation systems that integrate semantic information into the biomedical scientific literature in a standardized way.

\section{References}

1. Rebholz-Schuhmann D, Kirsch H, Couto F, Facts from text - is text mining ready to deliver? PLoS Biol 3(2):e65, 2005.

2. Miwa M, Sætre R, Kim J-D and Tsujii J, Event extraction with complex event classification using rich features, J Bioinform Comput Biol 8(1):131-146, 2010.

3. Sasaki Y, McNaught J, Ananiadou S, The value of an in-domain lexicon in genomics QA, J Bioinform Comput Biol 8(1):147-161, 2010.

4. Rebholz-Schuhmann D, Jimeno-Yepes A, van Mulligen E, Kang N, Kors J, Milward D, Corbett P, Buyko E, Beisswanger E, Hahn U, CALBC Silver Standard Corpus, J Bioinform Comput Biol 8(1):163-179, 2010. 